\title{
SOME REMARKS ON THE POLARIZATION OF 200-MC/S SOLAR RADIO EMISSION
}

\author{
M. H. COHEN \\ Cornell University, Ithaca, New York, U.S.A. \\ A. D. FOKKER \\ Ionosphere and Radio Astronomy Section, Netherlands Postal \\ and Telecommunications Services \\ The Hague, The Netherlands
}

1. LiNEAR POLARIZATION IN TYPE III BURSTS (M. H. COHEN)

3

Detecting a weak linearly polarized component in an isolated solar burst is very difficult. It is a unique event and it is not possible to make a series of observations to reduce the error. Interference and reflections confuse the result and it is extremely difficult to determine the equipment parameters precisely, especially those pertaining to the antenna.

We have, however, a number of unambiguous examples of weak linear (or highly elliptical) polarization in type III bursts, recorded with the narrow-band (10 to $18 \mathrm{kc} / \mathrm{s}) 200-\mathrm{Mc} / \mathrm{s}$ polarimeter at the Cornell Radio Observatory [1]. In some of these cases there are other unpolarized or weakly circularly polarized bursts nearby that act more or less as a control on calibration and antenna errors, and on ground reflections. A brief description of three of these events follows.

1. 1957 June $14,22^{\mathrm{h}} 04^{\mathrm{m}}$ U.T. (Fig. 1). This is a group of six small to

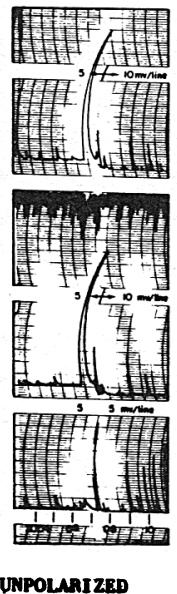

UNPOLARIZED

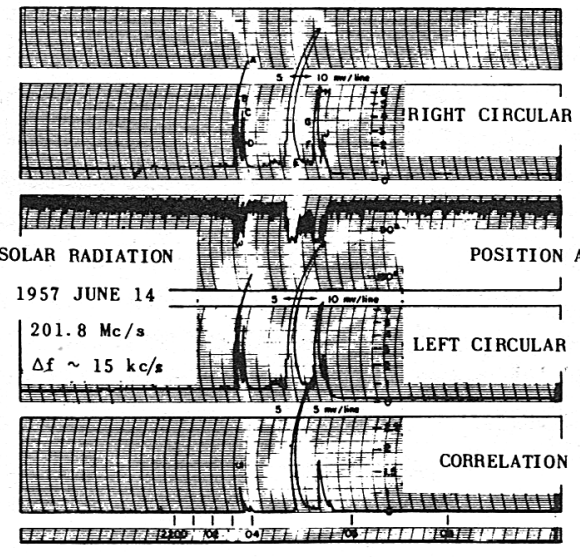

3OK LINEAR POLARIZATION
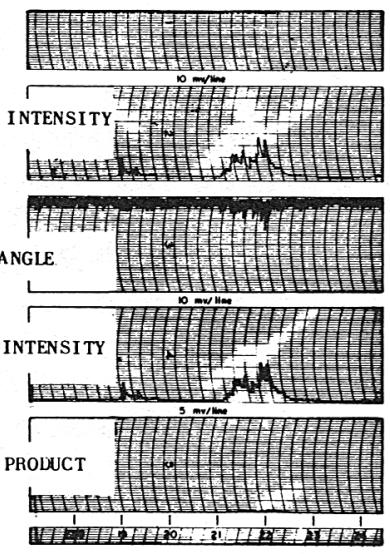

UNPOLARIZED

FIg. 1. Linear polarization of a group of type III bursts. 
medium bursts that are 30 per cent linearly polarized. At $20^{\mathrm{h}} 07^{\mathrm{m}}$ and $22^{\mathrm{h}} 22^{\mathrm{m}}$ there are unpolarized bursts of comparable magnitude with noticeably smaller correlator outputs, so the $22^{\mathrm{h}} 04^{\mathrm{m}}$-group has more polarization, in spite of calibration and antenna errors. The residual correlator signal at $20^{\mathrm{h}} 07^{\mathrm{m}}$ and $22^{\mathrm{h}} 22^{\mathrm{m}}$ is the noise due to the unpolarized radiation.

The bursts at $22^{\mathrm{h}} 04^{\mathrm{m}}$ are remarkably homogeneous: the degree of polarization is $0.31 \pm 0.035$, and the axial ratio is $0.02 \pm 0.02$, left-hand. The orientation is determined only for the two largest bursts; it is about 110 degrees for both and does not change appreciably for 0.3 . Systematic errors are thought to be no more than \pm 0.05 in degree of polarization and axial ratio, and possibly \pm 10 degrees in orientation.

Spectral data for these bursts were kindly supplied by Dr. A. Maxwell of the Fort Davis Radio Astronomy Station of Harvard College Observatory [2]. The bursts are all of spectral type III. There was no reported optical solar activity.

2. 1957 April $17,20^{\mathrm{h}} 20^{\mathrm{m}}$ to $21^{\mathrm{h}} 00^{\mathrm{m}}$ U.T. (Fig. 2). This "outburst plus second part " was time-associated with a flare of importance $3^{+}$near the east limb of the sun. The flare produced an S. I. D. and, apparently, a geomagnetic disturbance the following day. The flare began at $20^{\mathrm{h}} 00^{\mathrm{m}}$ and weak continuum radiation began shortly after. The large bursts from $20^{\mathrm{h}} 27^{\mathrm{m}}$ to $20^{\mathrm{h}} 30^{\mathrm{m}}$ are

SOLAR RADIATION 1957 APRIL $17 \quad 201.8 \mathrm{Mc} / \mathrm{s} \quad \Delta f \sim 15 \mathrm{kc} / \mathrm{s}$
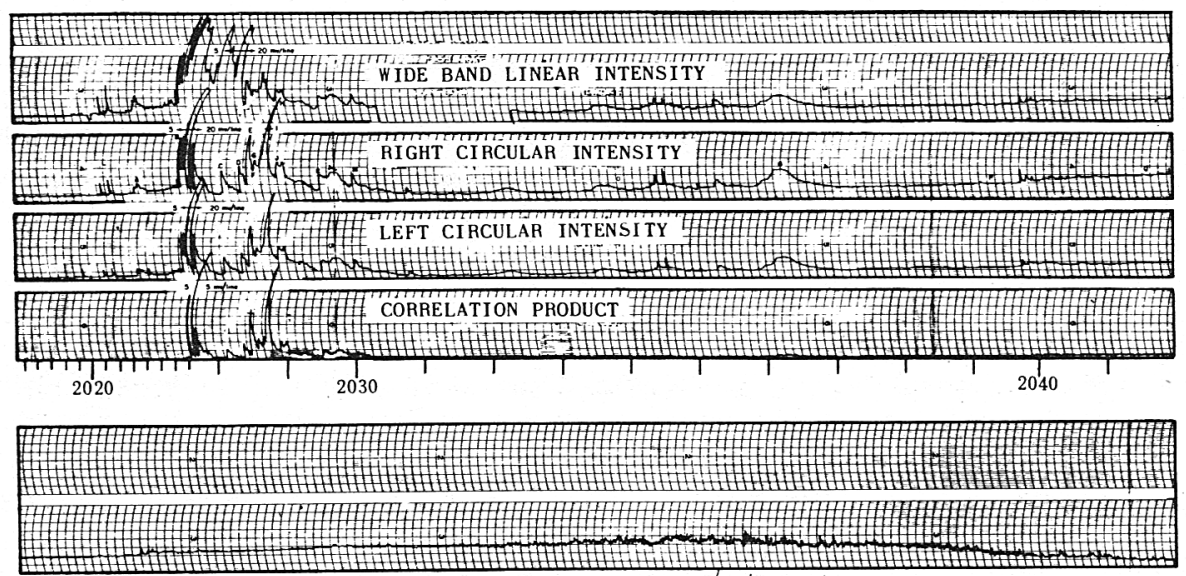

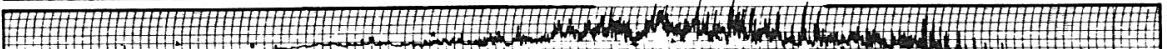
(1)

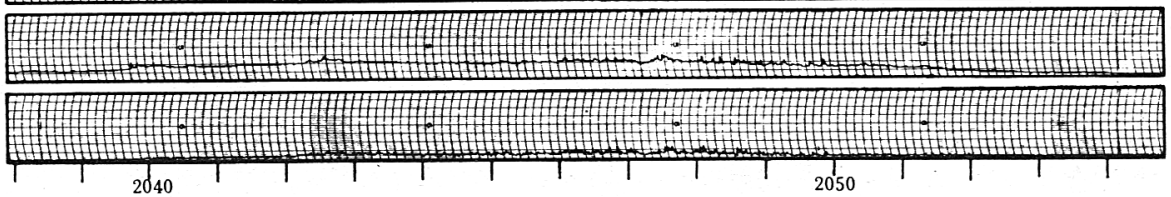

FIG. 2. Elliptical and circular polarization during "outburst plus second part." 
type III and are weakly elliptically polarized. The polarization varies among the bursts, being from 5 to 15 per cent, with axial ratios from zero to 0.8 , all right-hand. (The first few bursts at $20^{\mathrm{h}} 27^{\mathrm{m}}$ are relatively small and are either unpolarized or very weakly circularly polarized, left-hand.) The " second part" has a maximum at $20^{\mathrm{h}} 48^{\mathrm{m}}$; this is continuum radiation, but, according to Maxwell, it is not a good example of type IV radiation. The continuum is partially polarized, right circular; the degree of polarization tends to increase with time. At $20^{\mathrm{h}} 40^{\mathrm{m}}$ the radiation is 20 to 30 per cent polarized, and at $20^{\mathrm{h}} 50^{\mathrm{m}}$ it is 40 to 50 per cent polarized. The short storm-like bursts above the continuum from $20^{\mathrm{h}} 45^{\mathrm{m}}$ to $20^{\mathrm{h}} 52^{\mathrm{m}}$ are type $\mathrm{I}$ and are 70 to 100 per cent polarized, right circular.

The spectral data for this burst were also obtained from the Fort Davis Station. There was a type II burst following the group of type III bursts, but it was concentrated at frequencies below $200 \mathrm{Mc} / \mathrm{s}$ and is not prominent on our record.

3. 1958 March $21,19^{\mathrm{h}} 40^{\mathrm{m}}$ to $19^{\mathrm{h}} 46^{\mathrm{m}}$ U.T. There are three groups of bursts, all weakly elliptically polarized. The polarizations vary irregularly, from 15 to 25 per cent, with axial ratios from 0.2 to 0.7 , all right-hand. The orientation angle, however, appears to be almost constant for all the large bursts, over a period of several minutes. Spectral data for these bursts were kindly supplied by Professor F.T. Haddock and Dr. T. Takakura of the Observatory of the University of Michigan [3]. The first group is one large long complex burst. It has the type III fast-frequency drift but is unusual because it has such a long duration (one minute). The second group lasts two minutes and consists of about seven smaller bursts with durations 0.1 to $0 \mathrm{~m} 2$. These also have the fast-frequency drift but still are wider than the typical type III bursts. The third group lasts one minute and consists of many bursts with durations on the order of a few seconds. These are typical type III bursts.

There was no reported optical solar activity at this time.

\section{DIscussion}

Under the assumption of quasi-longitudinal propagation, the measurement of elliptical polarization allows one to deduce an upper limit to the Faraday rotation $\varphi$. The limit depends on receiver bandwidth. The 1957 June 14 bursts give $\varphi \leq 1.5 \times 10^{4}$ radians. This is on the order of the estimate made by Hatanaka [4] for a radial ray starting at $h=0.5 R_{\odot}$, with a dipole magnetic field having one gauss at the poles. The magnetic field can hardly be so smooth, but the limit still suggests either a weak field above the source or else a high source.

The constant parameters observed for the June 14 bursts more strongly suggest a high local source. The Faraday rotation will be very large near the plasma level and very minor changes in position would produce different orientations. The 1958 March 21 bursts also have the feature of constant orientation but the other parameters vary. The orientation was not measured for the April 17 outburst. 
Payne-Scott and Little [5] at $97 \mathrm{Mc} / \mathrm{s}$, saw two outbursts which had some linear polarization in their "second parts." These are different from the April 17 event; the frequency also is different. It is to be noted that the orientation was more or less constant during their outbursts. This is not stated explicitly but seems to follows from their remarks.

If there were a polarization-limiting region high in the corona it could easily explain the constant orientations. If the field there were nearly transverse the emergent radiation would be nearly linearly polarized and the orientation would be determined by this field, not by the source (which might be much deeper) nor by the propagation characteristics of the intervening corona. Exceptional geometric circumstances would be required, but these bursts are themselves exceptional.

We are indebted to A. Maxwell, and to F. T. Haddock and T. Takakura, for supplying the spectrum details of these bursts. The analysis of the March 21 event is due to H. Goldberg, who also has contributed to the data reduction techniques.

\section{POLARIZATION OF STORM RADIATION (A. D. FOKKER)}

Polarization measurements on $200-\mathrm{Mc} / \mathrm{s}$ solar radio emission have been made on a daily basis at the Nera observatory since November 1955 with equipment built by Mr. F. R. Neubauer. I should like to mention some features of polarization that result from our observations.

As is well known, enhanced solar radio emission and noise storms generally are circularly polarized. A point of interest is the question, how is the sense of polarization, right- or left-handed, related to properties of the sunspot region with which the source of enhanced radiation is associated? One would be justified in surmising that the strongest sunspot magnetic field will determine the sense of polarization. In general this is the field that belongs to the preceding sunspot of a bipolar group. The sense of polarization could be expected to be related to the hemisphere, north or south, where the noiseactive sunspot group is situated.

On the basis of a comparison of the sense of polarization at $97 \mathrm{Mc} / \mathrm{s}$ with the corresponding noise-active sunspots, it was concluded initially by the Australian workers, that the sense of polarization corresponds with the ordinary magneto-ionic component [6].

By interferometer-position determinations of solar sources of enhanced radiation at 169 and at $255 \mathrm{Mc} / \mathrm{s}$, which are obtained daily by the Nançay and Nera observatories, it is possible to indicate what sunspots have been noise-active on a particular day. In the majority of cases the same sunspots are designated as noise-active at these two frequencies. During the present sunspot maximum, sources of enhanced radiation often were associated with more than one sunspot region. A comparison of the hemisphere, north or south, where the noise-active sunspots are situated, with the sense of polarization of radio noise is possible whenever the hemisphere is unambiguously defined. This is the case when only one spot can be considered as noiseactive or when all noise-active sunspots are situated in the same hemisphere. 
On making this comparison we could find no correlation at all of the sense of polarization and the hemisphere. A northern noise-active sunspot has about equal chances of being accompanied by a source which produces rightor left-handed polarization.

Sometimes mixed polarization is observed, indicating the presence of sources that produce opposite polarizations (Fig. 3). In a few cases this mixed polarization was observed when the two or more noise-active sunspots were situated in the same hemisphere (for example, 1957 August 31/September 1). This observation illustrates the absence of a hemisphere dependence in a striking manner.

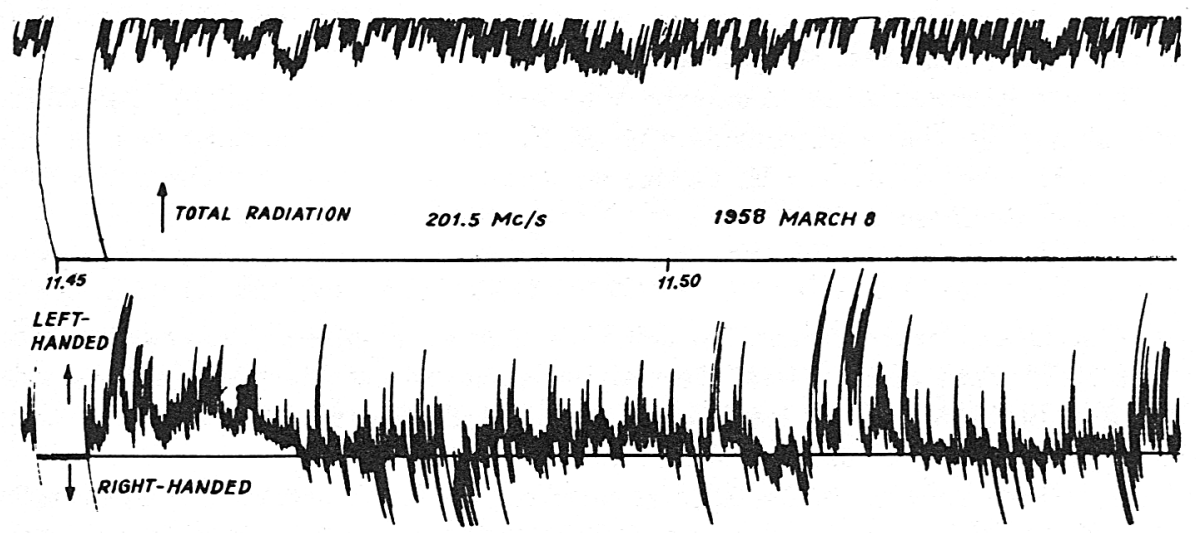

FIG. 3. Thoroughly mixed polarization during a noise storm.

Mixed polarization in general is observed when two or more sunspot regions contribute to the enhanced radiation. We noted one case of mixed polarization when only one sunspot group seemed to be noise-active (1956 December 19). This was a large bipolar sunspot group of the F-type. In view of the strong magnetic fields of both polarities which are commonly present in one or in several sunspot regions it is rather surprising that no more mixed polarization is observed than actually is the case.

A particular noise-active sunspot region generally gives rise to a constant sense of polarization throughout the period of noise production (a few days up to about one week).

In a minority of cases the enhanced radiation and the bursts are unpolarized. This seems to occur only when the level is slightly or moderately enhanced. Strongly enhanced radiation (level more than 4 times the quiet sun radiation) seems always to be polarized. Sources of unpolarized radiation were observed at various positions, both near the center and near the limb of the solar disk. On one occasion a source of enhanced radiation was observed near the western limb on two successive days (1956 June 25, 26). The source evidently was connected with a sunspot very close to the limb. The noise storms in progress on these two dates had very much the same character. However, on the first day the storm was unpolarized, the second day it was rather strongly right-handed polarized. 
Occasionally a noise storm at its beginning is weakly and, sometimes, irregularly polarized. It subsequently develops to a strongly uniformly polarized storm. This behavior has been observed both on the occasion of a very intense storm of long duration (1957 February 26) and on the occasions of some shorter duration isolated storms. The reverse

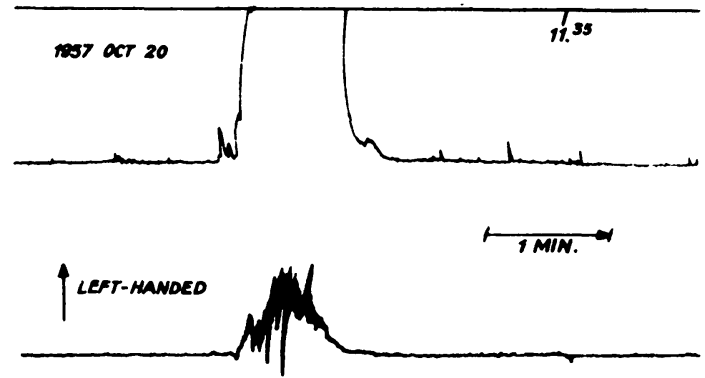

FIG. 4. Weakly but irregularly polarized outburst. case, of a storm's gradually becoming unpolarized, never has been observed.

In general the continuum and the stormbursts have almost identical degrees of polarization, often near 100 per cent. If the continuum has a smaller degree of polarization than the stormbursts there often is some mixed polarization, suggesting the presence of a source that contributes an oppositely polarized component to the continuum. In one case, when only one source seemed to be active, the continuum was hardly polarized whereas the bursts were strongly polarized (1958 March 12). The reverse, of the continuum's being polarized without the bursts' being polarized, was also observed once (1956 August 11).

The polarization characteristics of outbursts are very varied. Type IV outbursts may be both polarized or unpolarized. A certain category of large flare-associated outbursts initially are unpolarized or weakly irregularly polarized and become strongly and uniformly polarized after some 5 to 15 minutes. One big flare-associated outburst (1957 August 31) during the first three minutes consisted of a thoroughly mixed polarized noise storm with very short bursts.

Most type III bursts are unpolarized, but some of them present weak, sometimes irregular, circular polarization (Fig. 4). A series of bursts showing various polarizations is illustrated in Fig. 5.

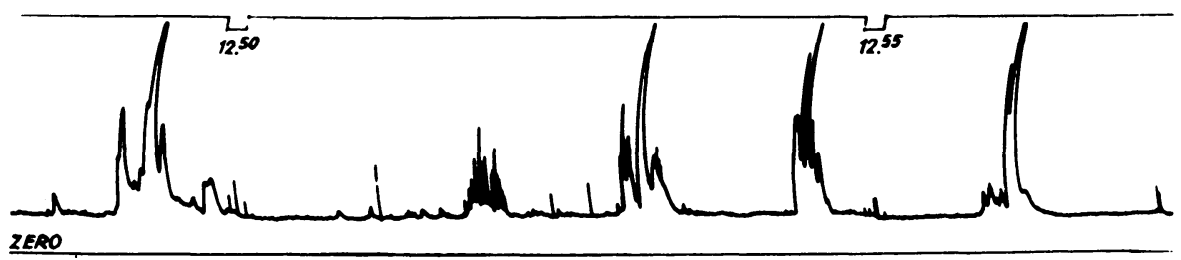

1958 JAN 6

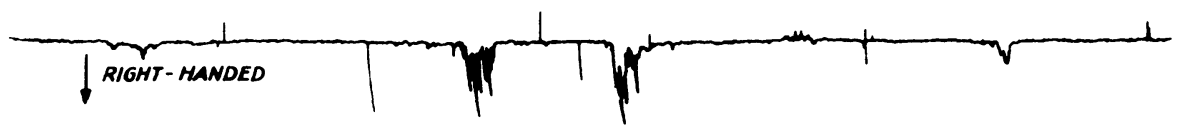

FIG. 5. Various polarizations during a series of bursts. 
My thanks are due to Mr. F. R. Neubauer for the design and the construction of the equipment and to the personnel of the Nera observatory (leader: $\mathrm{Mr}$. L. D. de Feiter) for the daily operation of the polarimeter.

\section{REFERENCES}

[1] Cohen, M. H. Proc. I.R.E. 46, 183, 1958.

[2] Private communication.

[3] Private communication.

[4] Hatanaka, T. Pub. Astr. Soc. Japan, 8, 73, 1956.

[5] Payne-Scott, R., and Little, A. G. Aust. J. Sci. Res., A 5, 32, 1952.

[6] Payne-Scott, R., and Little, A. G. Aust. J. Sci. Re8., A 4, 508, 1951.

\section{Discussion}

Takakura: Dr. Hatanaka sends me the following notes on the type I bursts observed on $200 \mathrm{Mc} / \mathrm{s}$ simultaneously by an interferometer and polarimeter at Tokyo: (1) The scatter of sizes of type I bursts ranged from less than 1 up to 5 minutes of arc; sometimes they were smaller than the area of the sunspot group, but sometimes larger. (2) Generally, there is no correlation between the polarization and the position in each active region, even when they are spread over a bipolar spot group. (3) The degree of polarization remains fairly constant during a whole transit across the solar disk, except at the extreme limb, but this limb effect is uncertain because of the decrease of activity at the limb.

Roberts: Komesaroff at Sydney has made a study of the polarization of the different spectral types (Aust. J. Phys. 11, 201, 1958). He found approximately 50 per cent of type III bursts were substantially polarized-usually approximately circular. I would like to ask whether Dr. Cohen has found cases of circular polarization in type III bursts?

Cohen: Strong circular polarization in type III bursts is rare. Weak circular polarization is common. 